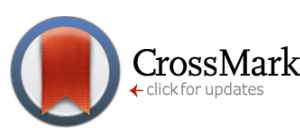

*For correspondence:

alesaleh70@yahoo.com

Competing interests: The authors declare that no competing interests exist.

Received: 29 October 2016 Accepted: 04 February 2017 Published: 22 February 2017

Copyright The Author(s) 2017. This article is published with open access by BioMedPress (BMP).

This article is distributed under the terms of the Creative Commons Attribution License (CC-BY 4.0) which permits any use, distribution, and reproduction in any medium, provided the original author(s) and the source are credited.

\section{The incidence and mortality of lip and oral cavity cancer and its relationship to the 2012 Human Development Index of Asia}

\author{
Amir Tiyuri ${ }^{1}$, Abdollah Mohammadian-Hafshejani' ${ }^{2}$, Elham Iziy ${ }^{3,4}$, \\ Hamidreza Sadeghi Gandomani ${ }^{5}$, Hamid Salehiniya ${ }^{6,7, *}$
}

\author{
1Department of Epidemiology and Biostatistics, School of Public Health, Tehran \\ University of Medical Sciences, Tehran, Iran \\ ${ }^{2}$ Department of Epidemiology and Biostatistics, School of Public Health, Isfahan \\ University of Medical Sciences, Isfahan, Iran \\ ${ }^{3}$ Traditional and Complementary Medicine Research Center, Sabzevar University of \\ Medical Sciences, Sabzevar, Iran \\ ${ }^{4}$ Department of Biology, Faculty of sciences, Islamic Azad University, Sciences and \\ Researches Branch, Tehran, Iran \\ ${ }^{5}$ Trauma Nursing Research Center, Faculty of Nursing and Midwifery, Kashan University of \\ Medical Sciences, Kashan, Iran \\ ${ }^{6}$ Zabol University of Medical Sciences, Zabol, Iran \\ ${ }^{7}$ Tehran University of Medical Sciences, Tehran, Iran
}

\section{Abstract}

Introduction: Lip and oral cavity cancer is one of the most prevalent cancers in Asia and considered to be a major public health problem due to the low survival rate. Because of the importance of access to information about this cancer (including incidence, mortality rate and relation to socioeconomic indicators), this study aims at investigating the incidence and mortality of lip and oral cavity cancer and its relationship with the Human Development Index (HDI) of Asia (from 2012). Method: This study was an ecological study in Asia for assessment of the correlation between age-specific incidence rate (ASIR) and age-specific mortality rate (ASMR) with the HDI and its components which include: life expectancy at birth, mean years of schooling and gross national income (GNI) per capita. Data on the standardized incidence ratio (SIR) and the standardized mortality ratio (SMR) for every Asian country for the year 2012 were obtained from the global cancer project and data on the HDI and its components were extracted from the World bank site. 
We used a bivariate method for assessment of the correlation between the SIR and SMR with the $\mathrm{HDI}$ and its individual components. Statistical significance was assumed if $P<0.05$. All reported P-values were two-sided. Statistical analyses were performed using SPSS (Version 15.0, SPSS Inc.). Results: A total incidence of 162,506 cases and 95,005 deaths were recorded in Asian countries in 2012. Countries with the highest SIR (per $100,000)$ were the following: Maldives (11), Sri Lanka (10.3), Pakistan (9.8), Bangladesh (9.4), and India (7.2). The highest SMR was observed in the following countries: Pakistan (5.9), Bangladesh (5.6), Afghanistan (5.1), India (4.9), and Maldives (4.1). The correlation between SIR of lip and oral cavity cancer and HDI was $-0.378(p=0.010)$, with life expectancy at birth at $-0.324(p=0.028)$, mean years of schooling at $-0.283(p=0.057)$, and level of income per each person of the population at $-0.279(p=0.060)$. Moreover, the correlation was $-0.664(p \leq 0.001)$ between SMR and HDI. Conclusion: A significant reverse correlation was seen between the incidence and mortality rate of lip and oral cavity cancer and the HDI in Asia. The incidence and mortality of this type of cancer was high in developing or less developed countries.

\section{Keywords}

Lip and oral cavity cancer, Human development index, Incidence, Mortality, Asia

\section{Introduction}

Cancers are among the leading causes of disease burden and mortality in the world and are regarded as a significant and growing public health problem around the world (Global Burden of Disease Cancer, 2015; Torre et al., 2015). Among cancers, lip and oral cavity cancer is the result of aggressive tumors originating from external lip and oral cavity and is the eighth most common cancer in men and the fourteenth most common cancer in women worldwide (de Camargo Cancela et al., 2010; Farah et al., 2014). In 2012, 14.1 million new cancer cases and 8.2 million cancer deaths occurred in the world; among them, 300,400 new cases and 145,400 deaths were due to lip and oral cavity cancer, accounting for more than $2 \%$ of new cases and $1.7 \%$ cases of death in the world, respectively (Torre et al., 2015). Most cancers of the lip and oral cavity have the same preventable risk factors (Warnakulasuriya, 2009). Smoking, alcohol, and chewing tobacco and betel quid (synergistic relationship) are the main risk factors for this type of cancer (Lin et al., 2005; Monteiro et al., 2013; Neville and Day, 2002; Warnakulasuriya, 2009). Poor eating habits, sun overexpsoure, viral infections (particularly human papillomavirus (HPV)), poor oral hygiene, and 
socio-economic factors are also important risk factors for lip and oral cavity cancers ( de Camargo Cancela et al., 2010; Farah et al., 2014; Funk et al., 2002; Ribeiro et al., 2015; Warnakulasuriya, 2009).

There is a large geographic variation in the incidence of lip and oral cavity cancer. The highest incidence rates have been reported from Malaysia, South Central Asia, and East and Central Europe, while the lowest rates have been reported from West Africa and East Asia. In recent decades, the incidence rate of lip and oral cavity cancers have decreased in men and women from Asia, North America and Australia, and in men from South and West Europe. However, the rates have increased in men and women from East and North Europe and in women from South and West Europe. The main reasons for this are the rising tobacco epidemic trend as well as the increased prevalence of HPV infection in some countries (Torre et al., 2015; Yako-Suketomo and Matsuda, 2010). Lip and oral cavity cancer is $90 \%$ squamous cell carcinoma and is often seen in middle-aged and older people. Its mortality is higher in men and black people, but lower in women (due to less exposure to risk factors such as smoking and alcohol) (de Camargo Cancela et al., 2010; Funk et al., 2002; Neville and Day, 2002; Yako-Suketomo and Matsuda, 2010).

Studies have shown that socioeconomic inequalities which affect behavior and lifestyle have a relation to the incidence and mortality rates of oral cavity cancer. However, some studies have shown conflicting results (Chen et al., 2009; de Camargo Cancela et al., 2010; Patel et al., 2012; Warnakulasuriya, 2009). To review countries' economic and social conditions, various indicators have been defined. One of the most important of the indicators is the Human Development Index (HDI) (Giebel et al., 2010; Hu et al., 2013). This index was first used by the United Nations Development Program and is a combination of three major factors- longevity, knowledge, and standard of living. The HDI is represented as a number between zero and one. Longevity is measured by life expectancy at birth and expressed as Life Expectancy Index. Knowledge is evaluated by a combination of adult literacy rate and the rate of enrollment at primary, secondary and tertiary schools (Education Index). The standard of living is measured by the Gross Domestic Product per capita, with purchasing power parity in US dollars (Gross Domestic Product Index) (Giebel et al., 2010; Hou et al., 2015; Rahi, 2011).

Some studies have shown the relationship between HDI and cancer incidence and mortality (Fidler et al., 2016; Pakzad et al., 2016; Rafiemanesh et al., 2016; Razi et al., 2016). However, to date, no study has been conducted to investigate the relationship between the HDI and the incidence and mortality of lip and oral cavity cancer in Asia. Knowledge of information about the incidence and mortality of lip and oral cavity cancer and its related factors can be useful for planning and developing policies related to health care. This study was aimed to determine the standardized incidence ratio (ASIR) and the standardized mortality ratio (SMR) of lip and oral cavity cancers, and the relationship of ASIR and ASMR with the $2012 \mathrm{HDI}$ of Asian countries. 


\section{Methods}

This study was an ecological study in Asia with the goal of assessing the correlation between age-specific incidence and mortality rates of lip and oral cavity cancer with the Human Development Index (HDI) and its components (life expectancy at birth, mean years of schooling, and gross national income per capita. Data about the age-specific incidence and mortality rates for every Asian country for the year 2012 were obtained from the global cancer project available online (http://globocan.iarc.fr/Default.aspx) (Ferlay J S, 2012). The HDI from the Human Development Report of 2013 (Malik, 2013) included information about the HDI and its components for every country in 2012.

A method of age-specific incidence and mortality rates from the global cancer project of the International Agency for Research on Cancer (France) was previously reported (Ferlay et al., 2014; Jemal et al., 2011; Torre et al., 2015).

\section{Human Development Index (HDI)}

The Human Development Index (HDI) is derived from a composite measure of indicators along three dimensions: life expectancy at birth, mean years of schooling and level of income per each person of the population (i.e. gross national income per capita) (Malik, 2013).

\section{Statistical analysis}

In this study, we used the correlation bivariate method for assessment of correlation between age-specific incidence and mortality rates with HDI and its components (including life expectancy at birth, mean years of schooling and gross national income per capita. Statistical significance was assumed if $\mathrm{P}<0.05$. All reported P-values were two-sided. Statistical analyses were performed using SPSS software (Version 15.0, SPSS Inc.).

\section{Results}

Overall, 162,506 cases of lip and oral cavity cancer were recorded in Asian countries in 2012. Of these cases, 106,308 (65.41\%) were men and 56,198 cases (34.58\%) were women. The sex ratio (male to female) was 1.89. The five countries with the highest number of new cases of lip and oral cavity cancer were:

1) India (77,002 cases),

2) China (21,413 cases),

3) Pakistan (12,761 cases), 
4) Bangladesh (10,550 cases),

5) Japan (8,306 cases).

These 5 countries, collectively, had a sum of 130,033 cases (80.01\%).

Of the Asian countries, the 5 countries with the highest standardized incidence rates of lip and oral cavity cancer were:

1) Maldives (standardized rate of 11 per 100,000 people),

2) Sri Lanka (10.3 per 100,000 people),

3) Pakistan (9.8 per 100,000 people),

4) Bangladesh (9.4 per 100,000 people),

5) India (7.2 per 100,000 people).

Conversely, the 5 countries with the lowest standardized rates of lip and oral cavity cancer were:

1) China (1.2 per 100,000 people),

2) Democratic Republic of Korea (1.3 per 100,000 people),

3) Kuwait (1.5 per 100,000 people),

4) Azerbaijan (1.7 per 100,000 people), and

5) Jordan (1.7 per 100,000 people).

The number as well as crude and standardized incidence rates of the cancer, according to sex, of the Asian countries are presented in Table 1. The countries are classified from highest to lowest, based on standardized incidence rates. The highest and lowest standardized incidence rates are indicated for both sexes (Table 1, Fig. 1).

On the other hand, 195,005 cases of death from of lip and oral cavity cancer have occurred in Asia in 2012. Of the cases, 62,860 (66.16\%) were men and 32,145 cases $(33.83 \%)$ were women. The sex ratio of death from lip and oral cavity cancer in Asian countries was 1.95. Of these, the largest numbers of deaths were seen in:

1) India (52,067 cases),

2) China (11,337 cases),

3) Pakistan (7,766 cases), 
4) Bangladesh (6,571 cases), and

5) Japan (3,994 cases).

These five countries, collectively, had a sum of 80,731 cases $(84.97 \%)$ of deaths.

Of the Asian countries, the 5 countries with the highest standardized mortality rates of lip and oral cavity cancer were:

1) Pakistan (5.9 per 100,000 people),

2) Bangladesh (5.6 per 100,000 people),

3) Afghanistan (5.1 per 100,000 people),

4) India (4.9 per 100,000 people), and

5) Maldives (4.1 per 100,000 people).

Conversely, the 5 countries with the lowest standardized mortality rates of lip and oral cavity cancer were:

1) Qatar (0.4 per 100,000 people),

2) Kuwait (0.4 per 100,000 people),

3) Bahrain (0.4 per 100,000 people),

4) Oman (0.4 per 100,000 people), and

5) United Arab Emirates (0.5 per 100,000 people).

The number as well as crude and standardized mortality rates of the cancer, according to sex, of the Asian countries are presented in Table 2. The countries are classified from highest to lowest, based on standardized mortality rates. The highest and lowest standardized mortality rates are indicated for both sexes (Table 2, Fig. 1).

\section{Assessing the relationship between standardized incidence rate and the Human Development Index}

Overall, a negative correlation of 0.378 was seen between the standardized incidence rate of lip and oral cavity cancer and the $\mathrm{HDI}$; the correlation was statistically significant $(P=0.010)$. A negative correlation was also seen between components of the $\mathrm{HDI}$ and the standardized incidence rate. Moreover, a negative correlation was seen when assessing the relationship of the standardized incidence rate to life expectancy at birth $(0.324 ; P=0.028)$, to mean age of education (0.283; $\mathrm{P}=0.057)$, and to level of income per person of the population (0.279; $\mathrm{P}=0.060)$. 
Table 1. Number, crude and standardized incidence rates of lip and oral cavity cancer in Asian countries in 2012 (sorted by age standardized incidence rates of both sexes from highest to lowest)

\begin{tabular}{|c|c|c|c|c|c|c|c|c|c|c|c|}
\hline \multicolumn{4}{|c|}{$\begin{array}{c}\text { Lip, oral cavity } \\
\text { Estimated incidence, all ages: } \\
\text { both sexes }\end{array}$} & \multicolumn{4}{|c|}{$\begin{array}{c}\text { Lip, oral cavity } \\
\text { Estimated incidence, all ages: } \\
\text { male }\end{array}$} & \multicolumn{4}{|c|}{$\begin{array}{c}\text { Lip, oral cavity } \\
\text { Estimated incidence, all ages: } \\
\text { female }\end{array}$} \\
\hline 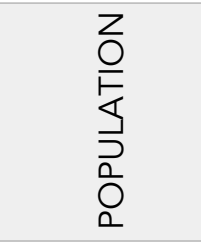 & $\begin{array}{l}\frac{n}{d} \\
\frac{0}{E} \\
\frac{\varrho}{\zeta}\end{array}$ & 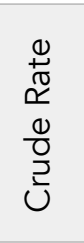 & $\sum_{\substack{\frac{1}{4} \\
\ll}}$ & 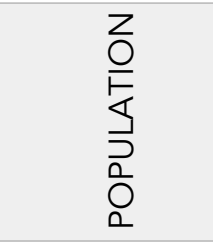 & $\begin{array}{l}\frac{n}{d} \\
\frac{0}{\varepsilon} \\
\frac{\varrho}{\zeta}\end{array}$ & 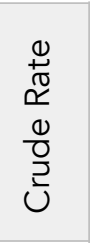 & $\sum_{\substack{\frac{1}{\infty} \\
\ll}}$ & 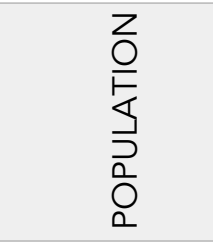 & $\begin{array}{l}\frac{n}{1} \\
\frac{\varrho}{\varepsilon} \\
\frac{\varrho}{\zeta}\end{array}$ & 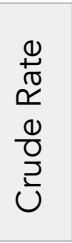 & $\sum_{\substack{\frac{1}{4} \\
\ll}}$ \\
\hline Maldives & 28 & 8.6 & 11.0 & Sri Lanka & 1845 & 17.6 & 15.4 & Pakistan & 5693 & 6.4 & 9.1 \\
\hline Sri Lanka & 2667 & 12.6 & 10.3 & Maldives & 20 & 12.2 & 15.4 & Brunei & 9 & 4.4 & 9.0 \\
\hline Pakistan & 12761 & 7.1 & 9.8 & Bangladesh & 7120 & 9.2 & 13.0 & Maldives & 8 & 5.0 & 6.4 \\
\hline Bangladesh & 10550 & 6.9 & 9.4 & Kazakhstan & 788 & 10.0 & 11.6 & Bangladesh & 3430 & 4.6 & 5.9 \\
\hline India & 77003 & 6.1 & 7.2 & Pakistan & 7068 & 7.7 & 10.5 & Sri Lanka & 822 & 7.6 & 5.7 \\
\hline Kazakhstan & 1083 & 6.6 & 6.3 & India & 53842 & 8.3 & 10.1 & Afghanistan & 435 & 2.7 & 5.4 \\
\hline Afghanistan & 1047 & 3.1 & 6.3 & Turkmenistan & 172 & 6.8 & 9.3 & Timor-Leste & 19 & 3.3 & 5.3 \\
\hline Myanmar & 2775 & 5.7 & 6.2 & Myanmar & 1810 & 7.5 & 8.6 & Cambodia & 304 & 4.1 & 5.2 \\
\hline Brunei & 18 & 4.4 & 6.0 & Nepal & 701 & 4.6 & 7.2 & India & 2316 & 3.8 & 4.3 \\
\hline Cambodia & 584 & 4.0 & 6.0 & Afghanistan & 612 & 3.5 & 7.1 & Lao PDR & 91 & 2.9 & 4.2 \\
\hline Timor-Leste & 40 & 3.4 & 5.6 & Cambodia & 280 & 3.9 & 7.1 & $\begin{array}{l}\text { State of } \\
\text { Palestine }\end{array}$ & 46 & 2.2 & 4.1 \\
\hline Turkmenistan & 224 & 4.3 & 5.6 & Armenia & 116 & 8.0 & 6.7 & Myanmar & 965 & 3.9 & 4.1 \\
\hline Nepal & 942 & 3.0 & 4.4 & Timor-Leste & 21 & 3.5 & 5.9 & Philippines & 1105 & 2.3 & 3.2 \\
\hline Kyrgyzstan & 163 & 3.0 & 4.0 & Kyrgyzstan & 108 & 4.0 & 5.8 & Thailand & 1551 & 4.4 & 3.0 \\
\hline Thailand & 3709 & 5.3 & 4.0 & Thailand & 2158 & 6.3 & 5.1 & Kazakhstan & 295 & 3.5 & 2.8 \\
\hline $\begin{array}{l}\text { State of } \\
\text { Palestine }\end{array}$ & 90 & 2.1 & 3.8 & Georgia & 159 & 7.8 & 4.9 & Malaysia & 363 & 2.5 & 2.8 \\
\hline Philippines & 2363 & 2.4 & 3.6 & Brunei & 9 & 4.3 & 4.5 & Kyrgyzstan & 55 & 2.0 & 2.5 \\
\hline Armenia & 165 & 5.3 & 3.6 & Bhutan & 14 & 3.5 & 4.3 & Turkmenistan & 52 & 2.0 & 2.4 \\
\hline Lao PDR & 140 & 2.2 & 3.4 & Uzbekistan & 425 & 3.0 & 4.2 & Yemen & 157 & 1.2 & 2.3 \\
\hline Bhutan & 19 & 2.5 & 3.2 & Philippines & 1258 & 2.6 & 4.1 & Nepal & 241 & 1.5 & 2.1 \\
\hline Malaysia & 776 & 2.6 & 3.0 & Japan & 4881 & 7.9 & 3.9 & Saudi Arabia & 164 & 1.3 & 2.1 \\
\hline Uzbekistan & 661 & 2.4 & 3.0 & Tajikistan & 75 & 2.2 & 3.7 & Israel & 116 & 3.0 & 2.0 \\
\hline
\end{tabular}




\begin{tabular}{|c|c|c|c|c|c|c|c|c|c|c|c|}
\hline Japan & 8306 & 6.6 & 2.9 & Singapore & 125 & 4.7 & 3.4 & Bhutan & 5 & 1.4 & 2.0 \\
\hline Tajikistan & 123 & 1.7 & 2.7 & $\begin{array}{l}\text { State of } \\
\text { Palestine }\end{array}$ & 44 & 2.0 & 3.4 & Japan & 3425 & 5.3 & 2.0 \\
\hline Georgia & 205 & 4.8 & 2.6 & Malaysia & 413 & 2.8 & 3.3 & Uzbekistan & 236 & 1.7 & 1.9 \\
\hline Singapore & 190 & 3.6 & 2.5 & Viet Nam & 1392 & 3.1 & 3.3 & Indonesia & 2327 & 1.9 & 1.9 \\
\hline Viet Nam & 2147 & 2.4 & 2.4 & Bahrain & 14 & 1.6 & 3.2 & Qatar & 3 & 0.6 & 1.9 \\
\hline Indonesia & 5329 & 2.2 & 2.3 & Iraq & 227 & 1.3 & 3.0 & Tajikistan & 48 & 1.3 & 1.8 \\
\hline Iraq & 411 & 1.2 & 2.3 & $\begin{array}{l}\text { Korea, } \\
\text { Republic of }\end{array}$ & 965 & 4.0 & 2.9 & $\begin{array}{l}\text { Iran, Islamic } \\
\text { Republic of }\end{array}$ & 617 & 1.7 & 1.8 \\
\hline Bahrain & 18 & 1.3 & 2.2 & Indonesia & 3002 & 2.5 & 2.8 & Mongolia & 18 & 1.2 & 1.8 \\
\hline $\begin{array}{l}\text { Korea, } \\
\text { Republic of }\end{array}$ & 1575 & 3.2 & 2.2 & Turkey & 939 & 2.5 & 2.7 & Iraq & 184 & 1.1 & 1.7 \\
\hline Israel & 231 & 3.0 & 2.2 & $\begin{array}{l}\text { Syrian Arab } \\
\text { Republic }\end{array}$ & 185 & 1.7 & 2.7 & Singapore & 65 & 2.5 & 1.7 \\
\hline Yemen & 283 & 1.1 & 2.2 & Lebanon & 54 & 2.6 & 2.6 & Oman & 12 & 1.0 & 1.7 \\
\hline $\begin{array}{l}\text { United Arab } \\
\text { Emirates }\end{array}$ & 80 & 1.0 & 2.1 & Lao PDR & 49 & 1.5 & 2.5 & $\begin{array}{l}\text { Korea, } \\
\text { Republic of }\end{array}$ & 610 & 2.5 & 1.6 \\
\hline Qatar & 23 & 1.2 & 2.1 & Israel & 115 & 3.0 & 2.4 & Viet Nam & 755 & 1.7 & 1.6 \\
\hline Turkey & 1502 & 2.0 & 2.1 & Azerbaijan & 97 & 2.1 & 2.4 & Lebanon & 37 & 1.7 & 1.5 \\
\hline $\begin{array}{l}\text { Iran, Islamic } \\
\text { Republic of }\end{array}$ & 1380 & 1.8 & 2.0 & $\begin{array}{l}\text { United Arab } \\
\text { Emirates }\end{array}$ & 59 & 1.1 & 2.4 & $\begin{array}{l}\text { United Arab } \\
\text { Emirates }\end{array}$ & 21 & 0.8 & 1.5 \\
\hline $\begin{array}{l}\text { Syrian Arab } \\
\text { Republic }\end{array}$ & 301 & 1.4 & 2.0 & $\begin{array}{l}\text { Iran, Islamic } \\
\text { Republic of }\end{array}$ & 763 & 2.0 & 2.2 & Turkey & 563 & 1.5 & 1.5 \\
\hline Saudi Arabia & 358 & 1.2 & 2.0 & Jordan & 47 & 1.4 & 2.2 & $\begin{array}{l}\text { Syrian Arab } \\
\text { Republic }\end{array}$ & 116 & 1.1 & 1.5 \\
\hline Lebanon & 91 & 2.1 & 2.0 & Mongolia & 19 & 1.4 & 2.2 & Armenia & 49 & 2.9 & 1.4 \\
\hline Mongolia & 37 & 1.3 & 2.0 & $\begin{array}{l}\text { Korea, } \\
\text { Democratic } \\
\text { Republic of }\end{array}$ & 283 & 2.3 & 2.1 & Kuwait & 9 & 0.8 & 1.3 \\
\hline Oman & 34 & 1.2 & 1.8 & Saudi Arabia & 194 & 1.2 & 2.0 & Jordan & 27 & 0.9 & 1.2 \\
\hline Jordan & 74 & 1.1 & 1.7 & Qatar & 20 & 1.4 & 2.0 & Bahrain & 4 & 0.8 & 1.2 \\
\hline Azerbaijan & 160 & 1.7 & 1.7 & Yemen & 126 & 1.0 & 2.0 & Azerbaijan & 63 & 1.3 & 1.1 \\
\hline Kuwait & 25 & 0.9 & 1.5 & Oman & 22 & 1.3 & 1.9 & Georgia & 46 & 2.0 & 0.9 \\
\hline $\begin{array}{l}\text { Korea, } \\
\text { Democratic } \\
\text { Republic of }\end{array}$ & 402 & 1.6 & 1.3 & China & 13656 & 1.9 & 1.6 & China & 7757 & 1.2 & 0.9 \\
\hline China & 21413 & 1.6 & 1.2 & Kuwait & 16 & 0.9 & 1.5 & $\begin{array}{l}\text { Korea, } \\
\text { Democratic } \\
\text { Republic of }\end{array}$ & 119 & 1.0 & 0.7 \\
\hline
\end{tabular}


In men, a negative correlation of 0.323 was seen between the standardized incidence rate of lip and oral cavity cancer and the $\mathrm{HDl}$; the correlation was statistically significant $(P=0.029)$. A negative correlation was also seen between components of the $\mathrm{HDI}$ and the standardized rate. Moreover, a negative correlation was seen when assessing the relationship of the standardized incidence rate to life expectancy at birth $(0.279 ; P=0.061)$, to mean age of education (0.167; $\mathrm{P}=0.267)$, and to level of income per person of the population (0.323; $P=0.029)$.

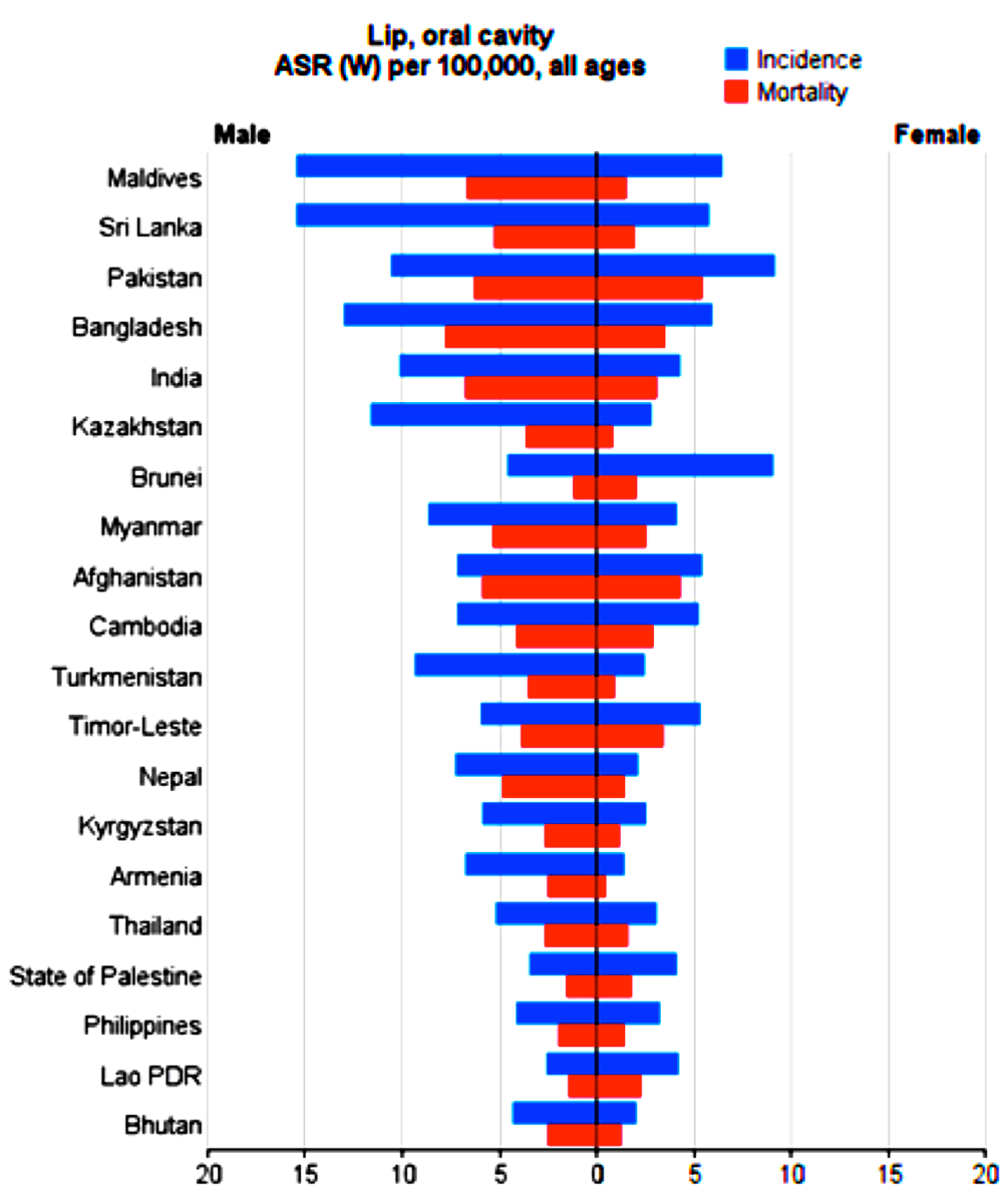

Figure 1. Standardized incidence and mortality rates of lip and oral cavity cancer in Asia in 2012 (extracted from GLOBOCAN 2012). 
In women, a negative correlation of 0.337 was seen between the standardized incidence rate of lip and oral cavity cancer and the $\mathrm{HDI}$; the correlation was statistically significant $(P=0.022)$. A negative correlation was also seen between components of the $\mathrm{HDI}$ and the standardized rate. Moreover, a negative correlation was seen when assessing the relationship of the standardized incidence rate to life expectancy at birth $(0.310 ; P=0.036)$, to mean age of education $(0.348, \mathrm{P}=0.018)$, and to level of income per person of the population (0.132; $P=0.382)$.

\section{Assessing the relationship between standardized mortality rate and the Human Development Index}

Overall, a negative correlation of 0.664 was seen between the standardized mortality rate of lip and oral cavity cancer and the $\mathrm{HDI}$; the correlation was statistically significant $(P \leq 0.001)$. Also, a significant negative correlation was seen between components of the HDI and the standardized rate. In fact, a negative correlation was seen when assessing the relationship of the standardized mortality rate to life expectancy at birth $(0.592 ; P \leq 0.001)$, to mean age of education (0.528; $P \leq 0.001)$, and to level of income per person of the population $(0.421 ; P=0.004)$.

In men, a negative correlation of 0.603 was seen between the standardized mortality rate of lip and oral cavity cancer and the HDI; the correlation was statistically significant $(P \leq 0.001)$. Also, a significant negative correlation was seen between components of the $\mathrm{HDI}$ and the standardized rate. In fact, a negative correlation was seen when assessing the relationship of the standardized mortality rate to life expectancy at birth $(0.518 ; P \leq 0.001)$, to mean age of education (0.448; $\mathrm{P}=0.002)$, and to level of income per person of the population (0.429; $P=0.003)$.

In women, a negative correlation of 0.666 was seen between the standardized mortality rate of lip and oral cavity cancer and the $\mathrm{HDI}$; the correlation was also statistically significant $(P \leq 0.001)$. Moreover, a significant negative correlation was seen between components of the HDI and the standardized rate. In fact, a negative correlation was seen when assessing the relationship of the standardized mortality rate to life expectancy at birth $(0.639 ; \mathrm{P} \leq 0.001)$, to mean age of education $(0.559 ; P \leq 0.001)$, and to level of income per person of the population (0.365; $\mathrm{P} \leq 0.001)$. 
Table 2. Number, crude and standardized mortality rates of lip and oral cavity cancer in Asian countries in 2012 (sorted by age standardized rates of both sexes from highest to lowest)

\begin{tabular}{|c|c|c|c|c|c|c|c|c|c|c|c|}
\hline \multicolumn{4}{|c|}{$\begin{array}{l}\text { Lip, oral cavity - Estimated mortality, } \\
\text { all ages: both sexes }\end{array}$} & \multicolumn{4}{|c|}{$\begin{array}{l}\text { Lip, oral cavity - Estimated } \\
\text { mortality, all ages: female }\end{array}$} & \multicolumn{4}{|c|}{$\begin{array}{l}\text { Lip, oral cavity - Estimated mortality, all } \\
\text { ages: male }\end{array}$} \\
\hline 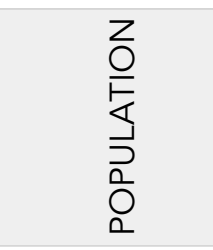 & $\begin{array}{l}\frac{n}{1} \\
\frac{0}{E} \\
\frac{1}{5} \\
Z\end{array}$ & 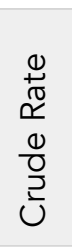 & $\underset{\frac{\alpha}{\infty}}{\sum_{\varangle}}$ & 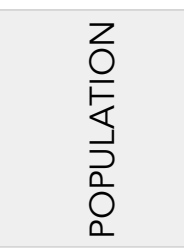 & $\begin{array}{l}\frac{\omega}{0} \\
\frac{0}{E} \\
\frac{\varrho}{z} \\
Z\end{array}$ & $\begin{array}{l}\underset{0}{+\infty} \\
\frac{+}{\alpha} \\
\frac{0}{0} \\
\frac{0}{2} \\
\frac{2}{U}\end{array}$ & 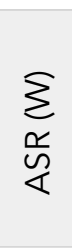 & 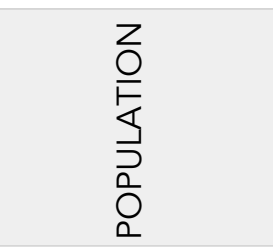 & $\begin{array}{l}\frac{\varrho}{d} \\
\frac{\varrho}{E} \\
\frac{\varrho}{Z}\end{array}$ & $\begin{array}{l}\stackrel{0}{+} \\
\frac{1}{\alpha} \\
\frac{0}{0} \\
\frac{0}{2} \\
\frac{1}{U}\end{array}$ & 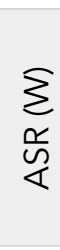 \\
\hline Pakistan & 7266 & 4.0 & 5.9 & Bangladesh & 4094 & 5.3 & 7.7 & Pakistan & 3220 & 3.6 & 5.4 \\
\hline Bangladesh & 6071 & 4.0 & 5.6 & India & 36436 & 5.6 & 6.7 & Afghanistan & 318 & 2.0 & 4.3 \\
\hline Afghanistan & 771 & 2.3 & 5.1 & Maldives & 8 & 4.9 & 6.6 & Bangladesh & 1977 & 2.6 & 3.5 \\
\hline India & 52067 & 4.1 & 4.9 & Pakistan & 4046 & 4.4 & 6.3 & Timor-Leste & 11 & 1.9 & 3.4 \\
\hline Maldives & 10 & 3.1 & 4.1 & Afghanistan & 453 & 2.6 & 5.8 & India & 15631 & 2.6 & 3.0 \\
\hline Myanmar & 1668 & 3.4 & 3.8 & Myanmar & 1090 & 4.5 & 5.3 & Cambodia & 165 & 2.2 & 2.9 \\
\hline Timor-Leste & 23 & 1.9 & 3.6 & Sri Lanka & 634 & 6.1 & 5.2 & Myanmar & 578 & 2.3 & 2.5 \\
\hline Sri Lanka & 916 & 4.3 & 3.5 & Nepal & 451 & 2.9 & 4.8 & Lao PDR & 48 & 1.5 & 2.3 \\
\hline Cambodia & 316 & 2.2 & 3.4 & Cambodia & 151 & 2.1 & 4.1 & Brunei & 2 & 1.0 & 2.0 \\
\hline Nepal & 606 & 2.0 & 2.9 & Timor-Leste & 12 & 2.0 & 3.8 & Sri Lanka & 282 & 2.6 & 1.9 \\
\hline Thailand & 1913 & 2.7 & 2.1 & Kazakhstan & 238 & 3.0 & 3.6 & State of Palestine & 17 & 0.8 & 1.7 \\
\hline Turkmenistan & 79 & 1.5 & 2.1 & $\begin{array}{l}\text { Turkmenist } \\
\text { an }\end{array}$ & 60 & 2.4 & 3.5 & Thailand & 799 & 2.2 & 1.6 \\
\hline Kazakhstan & 325 & 2.0 & 1.9 & Kyrgyzstan & 44 & 1.6 & 2.6 & Yemen & 99 & 0.8 & 1.6 \\
\hline Lao PDR & 74 & 1.2 & 1.9 & Thailand & 1114 & 3.2 & 2.6 & Maldives & 2 & 1.2 & 1.5 \\
\hline Bhutan & 10 & 1.3 & 1.8 & Bhutan & 7 & 1.8 & 2.4 & Nepal & 155 & 1.0 & 1.4 \\
\hline Kyrgyzstan & 69 & 1.3 & 1.8 & Armenia & 41 & 2.8 & 2.4 & Philippines & 451 & 0.9 & 1.4 \\
\hline $\begin{array}{l}\text { State of } \\
\text { Palestine }\end{array}$ & 35 & 0.8 & 1.6 & Philippines & 527 & 1.1 & 1.9 & Bhutan & 3 & 0.9 & 1.2 \\
\hline Philippines & 978 & 1.0 & 1.6 & Uzbekistan & 173 & 1.2 & 1.8 & Kyrgyzstan & 25 & 0.9 & 1.1 \\
\hline Yemen & 180 & 0.7 & 1.5 & Tajikistan & 34 & 1.0 & 1.7 & Turkmenistan & 19 & 0.7 & 0.9 \\
\hline Brunei & 4 & 1.0 & 1.4 & Mongolia & 14 & 1.0 & 1.7 & Mongolia & 8 & 0.6 & 0.9 \\
\hline Uzbekistan & 270 & 1.0 & 1.3 & Iraq & 107 & 0.6 & 1.5 & Iraq & 87 & 0.5 & 0.8 \\
\hline Tajikistan & 55 & 0.8 & 1.2 & Viet Nam & 632 & 1.4 & 1.5 & Uzbekistan & 97 & 0.7 & 0.8 \\
\hline Armenia & 58 & 1.9 & 1.2 & $\begin{array}{l}\text { State of } \\
\text { Palestine }\end{array}$ & 18 & 0.8 & 1.5 & Tajikistan & 21 & 0.6 & 0.8 \\
\hline
\end{tabular}




\begin{tabular}{|c|c|c|c|c|c|c|c|c|c|c|c|}
\hline Mongolia & 22 & 0.8 & 1.2 & Georgia & 49 & 2.4 & 1.4 & Indonesia & 987 & 0.8 & 0.8 \\
\hline Iraq & 194 & 0.6 & 1.1 & Japan & 2188 & 3.6 & 1.4 & Kazakhstan & 87 & 1.0 & 0.8 \\
\hline Viet Nam & 971 & 1.1 & 1.1 & Lao PDR & 26 & 0.8 & 1.4 & Malaysia & 97 & 0.7 & 0.8 \\
\hline Japan & 3994 & 3.2 & 1.1 & Yemen & 81 & 0.6 & 1.3 & Viet Nam & 339 & 0.7 & 0.7 \\
\hline Indonesia & 2250 & 0.9 & 1.0 & Malaysia & 156 & 1.0 & 1.2 & Japan & 1806 & 2.8 & 0.7 \\
\hline Malaysia & 253 & 0.9 & 1.0 & Indonesia & 1263 & 1.0 & 1.2 & Kuwait & 4 & 0.3 & 0.7 \\
\hline $\begin{array}{c}\text { Syrian Arab } \\
\text { Republic }\end{array}$ & 119 & 0.6 & 0.8 & Brunei & 2 & 1.0 & 1.1 & $\begin{array}{l}\text { Iran, Islamic } \\
\text { Republic of }\end{array}$ & 200 & 0.5 & 0.6 \\
\hline Georgia & 63 & 1.5 & 0.8 & $\begin{array}{c}\text { Syrian Arab } \\
\text { Republic }\end{array}$ & 73 & 0.7 & 1.1 & $\begin{array}{l}\text { Syrian Arab } \\
\text { Republic }\end{array}$ & 46 & 0.4 & 0.6 \\
\hline Singapore & 58 & 1.1 & 0.7 & Singapore & 41 & 1.5 & 1.1 & Saudi Arabia & 44 & 0.3 & 0.6 \\
\hline Turkey & 503 & 0.7 & 0.7 & $\begin{array}{c}\text { Korea, } \\
\text { Democratic } \\
\text { Republic of }\end{array}$ & 138 & 1.1 & 1.0 & Turkey & 187 & 0.5 & 0.5 \\
\hline $\begin{array}{l}\text { Iran, Islamic } \\
\text { Republic of }\end{array}$ & 449 & 0.6 & 0.7 & $\begin{array}{c}\text { Korea, } \\
\text { Republic of }\end{array}$ & 339 & 1.4 & 1.0 & Israel & 35 & 0.9 & 0.5 \\
\hline $\begin{array}{c}\text { Korea, Republic } \\
\text { of }\end{array}$ & 517 & 1.1 & 0.6 & Turkey & 316 & 0.9 & 0.9 & $\begin{array}{c}\text { United Arab } \\
\text { Emirates }\end{array}$ & 5 & 0.2 & 0.5 \\
\hline $\begin{array}{c}\text { Korea, } \\
\text { Democratic } \\
\text { Republic of }\end{array}$ & 200 & 0.8 & 0.6 & China & 7370 & 1.0 & 0.8 & Lebanon & 11 & 0.5 & 0.4 \\
\hline China & 11333 & 0.8 & 0.6 & Azerbaijan & 33 & 0.7 & 0.8 & Armenia & 17 & 1.0 & 0.4 \\
\hline Saudi Arabia & 97 & 0.3 & 0.6 & Lebanon & 16 & 0.8 & 0.8 & Jordan & 8 & 0.3 & 0.4 \\
\hline Israel & 70 & 0.9 & 0.6 & $\begin{array}{l}\text { Iran, Islamic } \\
\text { Republic of }\end{array}$ & 249 & 0.6 & 0.7 & China & 3963 & 0.6 & 0.4 \\
\hline Lebanon & 27 & 0.6 & 0.6 & Jordan & 15 & 0.5 & 0.7 & Singapore & 17 & 0.7 & 0.4 \\
\hline Jordan & 23 & 0.4 & 0.6 & Israel & 35 & 0.9 & 0.7 & Korea, Republic & 178 & 0.7 & 0.4 \\
\hline Azerbaijan & 54 & 0.6 & 0.6 & $\begin{array}{l}\text { Saudi } \\
\text { Arabia }\end{array}$ & 53 & 0.3 & 0.6 & Azerbaijan & 21 & 0.4 & 0.3 \\
\hline $\begin{array}{c}\text { United Arab } \\
\text { Emirates }\end{array}$ & 19 & 0.2 & 0.5 & Oman & 7 & 0.4 & 0.6 & $\begin{array}{c}\text { Korea, } \\
\text { Democratic } \\
\text { Republic of }\end{array}$ & 62 & 0.5 & 0.3 \\
\hline Oman & 8 & 0.3 & 0.4 & Qatar & 5 & 0.3 & 0.5 & Georgia & 14 & 0.6 & 0.3 \\
\hline Bahrain & 4 & 0.3 & 0.4 & Bahrain & 3 & 0.4 & 0.5 & Bahrain & 1 & 0.2 & 0.2 \\
\hline Kuwait & 8 & 0.3 & 0.4 & $\begin{array}{l}\text { United } \\
\text { Arab } \\
\text { Emirates }\end{array}$ & 14 & 0.2 & 0.5 & Oman & 1 & 0.1 & 0.1 \\
\hline Qatar & 5 & 0.3 & 0.4 & Kuwait & 4 & 0.2 & 0.3 & Qatar & 0 & 0.0 & 0.0 \\
\hline
\end{tabular}




\section{Discussion}

Although lip and oral cavity cancer accounts for less than 3\% of all cancer cases worldwide, its low survival rate and adverse consequences on quality of life have garnered it to be considered as a significant public health problem; in fact, two thirds of its burden occurs in developing countries (Costa et al., 2016; Farah et al., 2014; Global Burden of Disease Cancer, 2015; Ribeiro et al., 2015; Torre et al., 2015; Warnakulasuriya, 2009). Studies show that 162,506 new cases of lip and oral cavity cancer have been recorded in Asia in 2012, accounting for 56.1\% of all new cancer cases worldwide in 2012. There was a significant inverse relationship between the lip and oral cavity cancer and the HDI in Asia. The highest standardized incidence rates for this type of cancer, among the Asian countries, were seen in Maldives, Sri Lanka, Pakistan, Bangladesh and India, respectively. These countries were among the countries with medium HDI.

Since people who live in developing countries are exposed to a wider range of risk factors for cancer of the lip and oral cavity, the highest incidence rates are reported from these countries (Byakodi et al., 2012; de Camargo Cancela et al., 2010; Gupta et al., 2016; Rastogi et al., 2004). The most important risk factors of this cancer are tobacco use, alcohol, chewing tobacco, betel quid, poor eating habits, sun exposure, viral infections (especially HPV), and poor oral hygiene (de Camargo Cancela et al., 2010; Farah et al., 2014; Funk et al., 2002; Ribeiro et al., 2015; Warnakulasuriya, 2009). In India and Pakistan, about 100 million people use various types of smokeless tobacco and betel-quid chewing (Jayalekshmi et al., 2009). In addition to these countries, these tobacco and chewing habits are also common in Bangladesh, Afghanistan, Maldives, Sri Lanka and Nepal, which has led to an increased risk of lip and oral cavity cancers in these areas (Ariyawardana and Warnakulasuriya, 2011; Funk et al., 2002; Khan et al., 2016; Neville and Day, 2002; Sreeramareddy et al., 2014).

In the present study, an inverse relationship was seen between the incidence of lip and oral cavity cancer and the HDI components. The correlation was significant for life expectancy but insignificant for education and income. Studies have shown that the incidence of lip and oral cavity cancer is higher in people with lower education and income (de Camargo Cancela et al., 2010; Farah et al., 2014; Jayalekshmi et al., 2009; Johnson et al., 2010; Ribeiro et al., 2015; Swaminathan et al., 2009). People with less education are at greater risk of lip and oral cavity cancer due to less awareness of cancer risk factors, poor sanitary habits, greater consumption of alcohol and tobacco, and use of chewing tobacco (Gupta et al., 2016; Hashibe et al., 2003; Videnovic et al., 2016; Warnakulasuriya, 2009). Also, people with less income are more likely to have this type of cancer due to limited access to dental care, poor oral hygiene, consumption of fewer fruits and vegetables, greater HPV risk, and less protection against the sun (Arnold et al., 2016; Farah et al., 2014; Guha et al., 2007; Johnson et al., 2010; Monteiro et al., 2013; Morris et al., 2000; Pavia et al., 2006). Chen and colleagues also found an inverse relationship between income 
per capita and the incidence of lip and oral cavity cancer (Chen et al., 2009). In a systematic review and meta-analysis, done by Conway et al. on 41 case control studies from all around the world, economic and social conditions were found to be risk factors for oral cancer. These socioeconomic conditions included: low educational attainment ((odds ratio (OR): 1.85, 95\% confidence interval $(\mathrm{Cl})$ : 1.60-2.15)), low occupational social class (OR: 1.84, 95\% Cl: 1.47-2.31), and low income (OR: 2.41, 95\% Cl: 1.59-3.65) (Conway et al., 2008).

Based on the data from Asia, 95,005 deaths occurred due to lip and oral cavity cancer in 2012, which was equivalent to $66.9 \%$ of all cancer deaths in the world that year. A significant inverse relation was seen between lip and oral cavity cancer mortality and the HDI. Asian countries with the highest standardized mortality rate from lip and oral cavity cancer were Pakistan, Bangladesh, Afghanistan, India and Maldives, respectively. Afghanistan had low HDI while the rest had medium HDI. The findings showed a significant inverse relation between mortality from lip and oral cavity cancer and the HDI components (including life expectancy, education and income). Studies have shown that less education, lack of awareness about the symptoms of lip and oral cavity cancer, and delayed diagnosis are all factor which contribute to higher mortality rates (Albano et al., 2007; Kilander et al., 2001; Warnakulasuriya, 2009).

Despite advances in medical sciences, over the past several decades the overall five-year survival rate for lip and oral cavity cancer has not improved significantly, remaining at about 50-55\% (Neville and Day, 2002; Warnakulasuriya, 2009). In studies that were conducted in Asia, the overall five-year survival rate was $18 \%$ in Malaysia (Razak et al., 2010), 30.5 \% in India (Yeole et al., 2003), 52.8\% in Korea (Choi et al., 2014), and 61\% in Taiwan (Liu et al., 2010). Due to limited access to diagnostic and treatment services in low-income communities and to the high cost of services, people present with advanced stage lip and oral cavity cancer at the time of diagnosis. All the aforementioned are among the important reasons for the low 5-year survival of patients as well as the higher mortality rates in developing countries (Funk et al., 2002; Global Burden of Disease Cancer, 2015; Patel et al., 2012; Sargeran et al., 2008). McDonald et al. reported in their study that there was lower survival of head and neck cancers, including oral cavity cancer, in people of low socioeconomic statuses (McDonald et al., 2014).

Mortality and high burden of lip and oral cavity cancer, particularly in developing countries, continues to warrant public education. Awareness about the risk factors and symptoms of lip and oral cavity cancer, screening of high-risk groups, and planning for preventative measures can help the population most at risk for this kind of cancer and will be essential for effective prevention (de Camargo Cancela et al., 2010; Warnakulasuriya, 2009). 


\section{Conclusion}

In general, a significant inverse correlation was observed between the incidence of lip and oral cavity cancer and the HDI in Asia. Moreover, the incidence of this cancer was higher in developing countries. This correlation was also observed between cancer incidence and the HDI components; it was significant for life expectancy but insignificant for education and income. A significant inverse correlation was observed between deaths from lip and oral cavity cancer and the $\mathrm{HDI}$ and its components, and the mortality rate from this cancer was higher in developing countries.

\section{Abbreviations}

HDI: Human Development Index:

ASIR: Age-specific incidence rate

ASMR: Age-specific mortality rate

HPV: Human papillomavirus

\section{Acknowledgement}

Data on CANCER were obtained from the global cancer project and data on the HDI and its components were extracted from the World Bank site. Hereby we appreciate of the cooperation of all employees involved in data collection in the GLOBOCAN project and World Bank

\section{Author contribution}

All authors contributed to the design of the research. AMH, El and HSG collected the data. $\mathrm{AMH}, \mathrm{El}$ and $\mathrm{HS}$ conducted analysis and interpretation of data. All authors drafted the first version. HS, AT and AMH edited the first draft. All authors reviewed and commented on final draft. 


\section{References}

Albano, J.D., Ward, E., Jemal, A., Anderson, R., Cokkinides, V.E., Murray, T., Henley, J., Liff, J., and Thun, M.J. (2007). Cancer mortality in the United States by education level and race. Journal of the National Cancer Institute 99, 1384-1394.

Ariyawardana, A., and Warnakulasuriya, S. (2011). Declining oral cancer rates in Sri Lanka: are we winning the war after being at the top of the cancer league table? Oral diseases 17, 636-641.

Arnold, M., Renteria, E., Conway, D.I., Bray, F., Van Ourti, T., and Soerjomataram, I. (2016). Inequalities in cancer incidence and mortality across medium to highly developed countries in the twenty-first century. Cancer causes \& control : CCC 27, 999-1007.

Byakodi, R., Byakodi, S., Hiremath, S., Byakodi, J., Adaki, S., Marathe, K., and Mahind, P. (2012). Oral cancer in India: an epidemiologic and clinical review. Journal of community health 37, 316-319.

Chen, D.T., Chou, Y.F., Wu, H.P., Hsu, L.P., Wen, I.S., Lee, C.F., and Chen, P.R. (2009). Income and the incidence of oral cavity cancer: cross-national study. Journal of otolaryngology - head \& neck surgery = Le Journal d'oto-rhino-laryngologie et de chirurgie cervico-faciale 38, 208-211.

Choi, S.W., Moon, E.K., Park, J.Y., Jung, K.W., Oh, C.M., Kong, H.J., and Won, Y.J. (2014). Trends in the incidence of and survival rates for oral cavity cancer in the Korean population. Oral diseases 20, 773-779.

Conway, D.I., Petticrew, M., Marlborough, H., Berthiller, J., Hashibe, M., and Macpherson, L.M. (2008). Socioeconomic inequalities and oral cancer risk: a systematic review and meta-analysis of case-control studies. International journal of cancer 122, 2811-2819.

Costa, N.L., Goncalves, A.S., Martins, A.F., Arantes, D.A., Silva, T.A., and Batista, A.C. (2016). Characterization of dendritic cells in lip and oral cavity squamous cell carcinoma. Journal of oral pathology \& medicine : official publication of the International Association of Oral Pathologists and the American Academy of Oral Pathology 45, 418-424.

de Camargo Cancela, M., Voti, L., Guerra-Yi, M., Chapuis, F., Mazuir, M., and Curado, M.P. (2010). Oral cavity cancer in developed and in developing countries: populationbased incidence. Head \& neck 32, 357-367.

Farah, C.S., Simanovic, B., and Dost, F. (2014). Oral cancer in Australia 1982-2008: a growing need for opportunistic screening and prevention. Australian dental journal 59, 349-359.

Ferlay J S, E.M., Dikshit R, Eser S, Mathers C, Rebelo M, et al. (2012). GLOBOCAN 2012 v1.0, Cancer Incidence and Mortality Worldwide: IARC CancerBase No. 11. Lyon, France: International Agency for Research on Cancer; 2013. Available from: http:// globocan.iarc.fr. Accessed June 7, 2015.

Ferlay, J., Soerjomataram, I., Ervik, M., Dikshit, R., Eser, S., Mathers, C., Rebelo, M., Parkin, D., Forman, D., and Bray, F. (2014). GLOBOCAN 2012 v1. 0, Cancer Incidence and Mortality Worldwide: IARC CancerBase No. 11 [Internet]. 2013, Lyon, France: International Agency for Research on Cancer. globocan iarc fr/Default aspx. 
Fidler, M.M., Soerjomataram, I., and Bray, F. (2016). A global view on cancer incidence and national levels of the human development index. International journal of cancer $139,2436-2446$

Funk, G.F., Karnell, L.H., Robinson, R.A., Zhen, W.K., Trask, D.K., and Hoffman, H.T. (2002). Presentation, treatment, and outcome of oral cavity cancer: a National Cancer Data Base report. Head \& neck 24, 165-180.

Giebel, S., Labopin, M., Ehninger, G., Beelen, D., Blaise, D., Ganser, A., Bacigalupo, A., Czerw, T., Holowiecki, J., Fagundes, E.M., et al. (2010). Association of Human Development Index with rates and outcomes of hematopoietic stem cell transplantation for patients with acute leukemia. Blood 116, 122-128.

Global Burden of Disease Cancer, C. (2015). The Global Burden of Cancer 2013. JAMA oncology 1, 505-527.

Guha, N., Boffetta, P., Wunsch Filho, V., Eluf Neto, J., Shangina, O., Zaridze, D., Curado, M.P., Koifman, S., Matos, E., Menezes, A., et al. (2007). Oral health and risk of squamous cell carcinoma of the head and neck and esophagus: results of two multicentric casecontrol studies. American journal of epidemiology 166, 1159-1173.

Gupta, B., Johnson, N.W., and Kumar, N. (2016). Global Epidemiology of Head and Neck Cancers: A Continuing Challenge. Oncology 91, 13-23.

Hashibe, M., Jacob, B.J., Thomas, G., Ramadas, K., Mathew, B., Sankaranarayanan, R., and Zhang, Z.F. (2003). Socioeconomic status, lifestyle factors and oral premalignant lesions. Oral oncology 39, 664-671.

Hou, J., Walsh, P.P., and Zhang, J. (2015). The dynamics of Human Development Index. The Social Science Journal 52, 331-347.

Hu, Q.D., Zhang, Q., Chen, W., Bai, X.L., and Liang, T.B. (2013). Human development index is associated with mortality-to-incidence ratios of gastrointestinal cancers. World journal of gastroenterology 19, 5261-5270.

Jayalekshmi, P.A., Gangadharan, P., Akiba, S., Nair, R.R.K., Tsuji, M., and Rajan, B. (2009). Tobacco chewing and female oral cavity cancer risk in Karunagappally cohort, India. British Journal of Cancer 100, 848-852.

Jemal, A., Bray, F., Center, M.M., Ferlay, J., Ward, E., and Forman, D. (2011). Global cancer statistics. CA: a cancer journal for clinicians 61, 69-90.

Johnson, S., McDonald, J.T., Corsten, M., and Rourke, R. (2010). Socio-economic status and head and neck cancer incidence in Canada: a case-control study. Oral oncology 46, 200-203.

Khan, Z., Khan, S., Christianson, L., Rehman, S., Ekwunife, O., and Samkange-Zeeb, F. (2016). Smokeless tobacco and oral potentially malignant disorders in South Asia: a protocol for a systematic review. Systematic reviews 5, 142.

Kilander, L., Berglund, L., Boberg, M., Vessby, B., and Lithell, H. (2001). Education, lifestyle factors and mortality from cardiovascular disease and cancer. A 25-year followup of Swedish 50-year-old men. International journal of epidemiology 30, 1119-1126.

Lin, Y.S., Jen, Y.M., Wang, B.B., Lee, J.C., and Kang, B.H. (2005). Epidemiology of oral cavity cancer in taiwan with emphasis on the role of betel nut chewing. ORL; journal for oto-rhino-laryngology and its related specialties 67, 230-236.

Liu, S.Y., Lu, C.L., Chiou, C.T., Yen, C.Y., Liaw, G.A., Chen, Y.C., Liu, Y.C., and Chiang, W.F. (2010). Surgical outcomes and prognostic factors of oral cancer associated with betel quid chewing and tobacco smoking in Taiwan. Oral oncology 46, 276-282. 
Malik, K. (2013). Human development report 2013: The rise of the South. Human Progress in a Diverse World United Nations Development Programme, New York Available at http://hdr.undp.org/en/content/human-development-report-2013 (accessed 290713). 2013.

McDonald, J.T., Johnson-Obaseki, S., Hwang, E., Connell, C., and Corsten, M. (2014). The relationship between survival and socio-economic status for head and neck cancer in Canada. Journal of Otolaryngology - Head \& Neck Surgery 43, 1-6.

Monteiro, L.S., Antunes, L., Bento, M.J., and Warnakulasuriya, S. (2013). Incidence rates and trends of lip, oral and oro-pharyngeal cancers in Portugal. Journal of oral pathology \& medicine : official publication of the International Association of Oral Pathologists and the American Academy of Oral Pathology 42, 345-351.

Morris, R.E., Mahmeed, B.E., Gjorgov, A.N., Jazzaf, H.G., and Rashid, B.A. (2000). The epidemiology of lip, oral cavity and pharyngeal cancers in Kuwait 1979-1988. The British journal of oral \& maxillofacial surgery 38, 316-319.

Neville, B.W., and Day, T.A. (2002). Oral cancer and precancerous lesions. CA Cancer J Clin 52, 195-215.

Pakzad, R., Mohammadian-Hafshejani, A., Khosravi, B., Soltani, S., Pakzad, I., Mohammadian, M., Salehiniya, H., and Momenimovahed, Z. (2016). The incidence and mortality of esophageal cancer and their relationship to development in Asia. Annals of translational medicine 4, 29.

Patel, A.R., Prasad, S.M., Shih, Y.C., and Eggener, S.E. (2012). The association of the human development index with global kidney cancer incidence and mortality. The Journal of urology 187, 1978-1983.

Pavia, M., Pileggi, C., Nobile, C.G., and Angelillo, I.F. (2006). Association between fruit and vegetable consumption and oral cancer: a meta-analysis of observational studies. The American journal of clinical nutrition 83, 1126-1134.

Rafiemanesh, H., Mehtarpour, M., Khani, F., Hesami, S.M., Shamlou, R., Towhidi, F., Salehiniya, H., Makhsosi, B.R., and Moini, A. (2016). Epidemiology, incidence and mortality of lung cancer and their relationship with the development index in the world. Journal of thoracic disease 8, 1094-1102.

Rahi, M. (2011). Human development report 2010: Changes in parameters and perspectives. Indian journal of public health 55, 272-275.

Rastogi, T., Hildesheim, A., and Sinha, R. (2004). Opportunities for cancer epidemiology in developing countries. Nature reviews Cancer 4, 909-917.

Razak, A.A., Saddki, N., Naing, N.N., and Abdullah, N. (2010). Oral cancer survival among Malay patients in Hospital Universiti Sains Malaysia, Kelantan. Asian Pacific journal of cancer prevention : APJCP 11, 187-191.

Razi, S., Ghoncheh, M., Mohammadian-Hafshejani, A., Aziznejhad, H., Mohammadian, M., and Salehiniya, H. (2016). The incidence and mortality of ovarian cancer and their relationship with the Human Development Index in Asia. ecancermedicalscience 10.

Ribeiro, I.L.A., Medeiros, J.J.d., Rodrigues, L.V., Valença, A.M.G., and Lima Neto, E.d.A. (2015). Factors associated with lip and oral cavity cancer. Revista Brasileira de Epidemiologia 18, 618-629.

Sargeran, K., Murtomaa, H., Safavi, S.M., Vehkalahti, M.M., and Teronen, O. (2008). Survival after diagnosis of cancer of the oral cavity. The British journal of oral \& maxillofacial surgery 46, 187-191. 
Sreeramareddy, C.T., Pradhan, P.M., Mir, I.A., and Sin, S. (2014). Smoking and smokeless tobacco use in nine South and Southeast Asian countries: prevalence estimates and social determinants from Demographic and Health Surveys. Population health metrics $12,22$.

Swaminathan, R., Selvakumaran, R., Vinodha, J., Ferlay, J., Sauvaget, C., Esmy, P.O., Shanta, V., and Sankaranarayanan, R. (2009). Education and cancer incidence in a rural population in south India. Cancer epidemiology 33, 89-93.

Torre, L.A., Bray, F., Siegel, R.L., Ferlay, J., Lortet-Tieulent, J., and Jemal, A. (2015). Global cancer statistics, 2012. CA: A Cancer Journal for Clinicians 65, 87-108.

Videnovic, G., Illic, D., Miljus, D., Krasic, D., Vlahovic, Z., Zivkovic, S., and Pavlovic, A. (2016). Lip, oral cavity and pharyngeal cancers in the population of the city of Belgrade in the period 1999-2010. Vojnosanitetski pregled 73, 53-58.

Warnakulasuriya, S. (2009). Global epidemiology of oral and oropharyngeal cancer. Oral oncology 45, 309-316.

Yako-Suketomo, H., and Matsuda, T. (2010). Comparison of time trends in lip, oral cavity and pharynx cancer mortality (1990-2006) between countries based on the WHO mortality database. Japanese journal of clinical oncology 40, 1118-1119.

Yeole, B.B., Ramanakumar, A.V., and Sankaranarayanan, R. (2003). Survival from oral cancer in Mumbai (Bombay), India. Cancer causes \& control : CCC 14, 945-952. 